\title{
Preparation of Nano-Lignin as Antistatic Additive for Thermoplastic Polymers
}

\author{
Mertcan Özel ${ }^{1}$, Fatma Demir ${ }^{1}$, Bilge Baytekin ${ }^{1,2 *}$ \\ ${ }^{1}$ Bilkent University, Faculty of Science, Department of Chemistry 06800, Ankara, TURKEY \\ ${ }^{2}$ UNAM, Bilkent University, 06800, Ankara, TURKEY \\ * The corresponding author e-mail: b-baytekin@fen.bilkent.edu.tr
}

Keywords: ball-milling; biomass; contact electrification; mechanochemistry; static electricity.

Lignin is the second most abundant polymer in the plants after the cellulose counterpart. Only the general structure of the lignin can be shown which contains polyphenolic units in the amorphous cross-linked polymer chain.

Static electricity is a challenge that causes millions of dollars costs extensively in industries such as polymer manufacturing, space and pharmaceutical due to the detrimental effects of electrostatic discharge of accumulated charges on surface of the materials. Doping of the materials, for instance; polymers, with antistatic agents (additives) can prevent those problems nevertheless, the usage of additives can be solved the issue by increasing the conductivity of the final material, polymer or composite in order to remove the surface charges and surface electrification via either directly doping with conductive materials, or doping by additives that can increase the surface humidity of the final material.

Those doped materials usually lose their characteristic mechanical properties due to the high concentrations of the additive. The main mechanism of static charge formation should be wellunderstood in order to prevent challenges for the properties of the materials and because of the bond-breakages on the surface of the polymer materials, which causes mechanoradical active ends.

We have shown that by removing the mechanoradicals with antioxidants one can destabilize the charges and makes the materials antistatic. In this work, we extracted and characterized nano-lignin materials obtained from different bio-sources and propose to use the nano-lignin, which itself has antioxidant properties can be a good candidate as an antistatic agent for common thermoplastic polymers.

\section{Acknowledgements}

We acknowledge financial support from TÜBİTAK project number $\mathbf{1 1 6 Z 5 2 3 . ~}$ 